\title{
SLE retinopathy: evaluation by fluorescein angiography
}

\author{
J. G. LANHAM, T. BARRIE, E. M. KOHNER, AND G. R. V. HUGHES \\ From the Rheumatology and Medical Ophthalmology Units, Department of Medicine, Hammersmith Hospital, \\ London
}

SUMMARY Fifty-two patients with systemic lupus erythematosus (SLE) were examined by fluorescein angiography, and retinopathy was detected in 15 . Three patterns of retinopathy were discerned: 4 patients had disc vasculitis, 6 had multiple cotton-wool spots, and 5 had a normal fundal appearance but fluorescein leakage on angiography. One patient had arterial occlusive disease with retinal neovascularisation and another had extensive venous disease. With 3 exceptions retinopathy was found only in patients with active SLE. No association was discovered between retinopathy and cerebral disease; in particular, fluorescein angiography did not assist the diagnosis of mild cerebral lupus.

Structural similarities between retinal and cerebral blood vessels suggest that retinal vascular involvement in systemic lupus erythematosus (SLE) might mirror disease in the cerebral vessels. Fluorescein angiography is a useful means of demonstrating retinal microvascular abnormalities and has been proposed as a method of assessing suspected cerebral lupus. ${ }^{1}$

The assessement of disease activity in SLE is frequently difficult, and evaluation of cerebral disease can be particularly problematic. ${ }^{2}$ Neuropsychiatric manifestations of SLE are protean, but none of the investigative techniques currently employed are reliable in defining the presence and severity of cerebral disease. ${ }^{3}$ Many patients have episodes of depression or minor personality change while their disease is active without any diagnostic features of cerebral lupus. ${ }^{4}$ This suggests that subclinical cerebral involvement may occur, but no method of investigation is specific enough to detect it. Recently it has even been proposed that a chronic subclinical cerebritis may be common in SLE, producing a gradual decline in intellectual function. ${ }^{5}$

The significance of retinopathy in patients with systemic lupus erythematosus is not clearly established; the relationship of retinopathy with disease activity and cerebral disease has not previously received systematic study. The present study employed fluorescein angiography, colour photography, and

Accepted for publication 18 August 1981.

Correspondence to Dr J. G. Lanham, Hammersmith Hospital, Ducane Road, London W12 0HS. conventional examination of the fundus to investigate possible parallels between retinal vasculitis and CNS disease. Fundal abnormalities were related both to general disease activity and to the presence of cerebral disease at the time of ocular examination.

\section{Patients and methods}

Fifty-two patients with SLE were examined between 1973 and 1980 by means of colour photography of the retina and fluorescein angiography. All of them satisfied the preliminary ARA criteria for the classification of SLE. The majority were examined while in hospital under the care of the Rheumatology Unit. This examination was included in the protocol for the investigation of all patients, whether they were new patients admitted for initial assessment or old patients admitted because of disease exacerbations.

Table 1 The relationship between the activity of the SLE and cerebral disease at the time of ophthalmological examination and the 3 forms of retinopathy

\begin{tabular}{llllll}
\hline & & Total & $\begin{array}{l}\text { Disc } \\
\text { vasculitis }\end{array}$ & $\begin{array}{l}\text { Cotton-wool } \\
\text { spots }\end{array}$ & $\begin{array}{l}\text { Fluorescein } \\
\text { leakage }\end{array}$ \\
& & $(n=50)$ & $(n=4)$ & $(n=6)$ & $(n=5)$ \\
\hline $\begin{array}{llllll}\text { Disease } \\
\text { activity }\end{array}$ & $\begin{array}{l}\text { Inactive } \\
\text { Mild }\end{array}$ & 11 & 1 & 0 & 0 \\
& activity & 13 & 0 & 0 & 2 \\
& Active & 26 & 3 & 6 & 3 \\
\hline $\begin{array}{l}\text { Cerebral } \\
\text { disease }\end{array}$ & None & 34 & 3 & 4 & 4 \\
& Mild & 7 & 1 & 1 & 0 \\
\hline
\end{tabular}




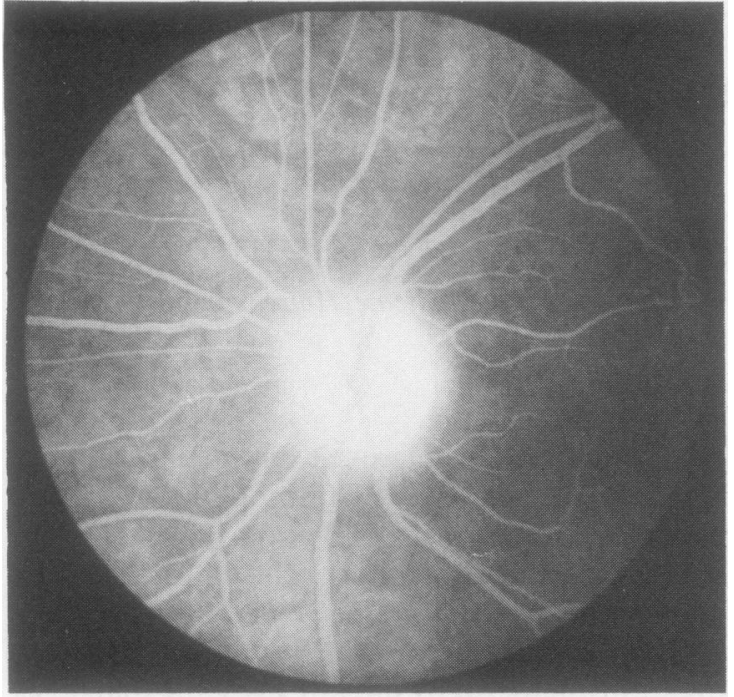

Fig. 1 Disc vasculitis. Fluorescein angiogram showing diffuse leakage of dye from the inflammed disc vessels.

The only bias in patient selection was the predominance of patients towards the more active end of the disease spectrum; there was no specific selection of patients with renal or cerebral disease. Treatment appropriate to the patients' clinical state had usually been instituted at the time of examination; the majority of patients were on steroids.

The patients were allocated to one of 3 groups according to a clinical assessment of disease activity. The inactive group comprised those patients having no signs or symptoms of active disease. The active group had clinically obvious arthritis, nephritis, cerebral disease, or extensive cutaneous vasculitis. The intermediate group had evidence of mild disease activity. Clinical features in these patients included arthralgias, minor rash, pleuritic pain, alopecia, and general malaise.

Patients were also placed in one of 3 groups on the basis of cerebral disease activity. Active cerebral disease was diagnosed if psychosis, seizures, organic brain syndrome, or focal neurological deficit was present. The evaluation of the clinicians caring for the patient at the time of the ophthalmological examination was accepted. This was invariably based not only on clinical parameters but also on other investigations such as cerebrospinal fluid (CSF) examination, electroencephalograph (EEG), and brain scan. The intermediate group had psychiatric disturbances without psychotic features; these included depressive and anxiety states and minor personality change. These patients are regarded in this study as having mild cerebral disease. Renal disease was diagnosed on the basis of impairment of renal function, haematuria, proteinuria (exceeding $500 \mathrm{mg}$ per day), an active urinary sediment, or an abnormal renal biopsy.

With 2 exceptions hypertensive and diabetic patients were not excluded from the study. The 2 patients who were excluded had hypertension and diabetes of sufficient severity and duration to account for their retinopathy, which was characteristic of
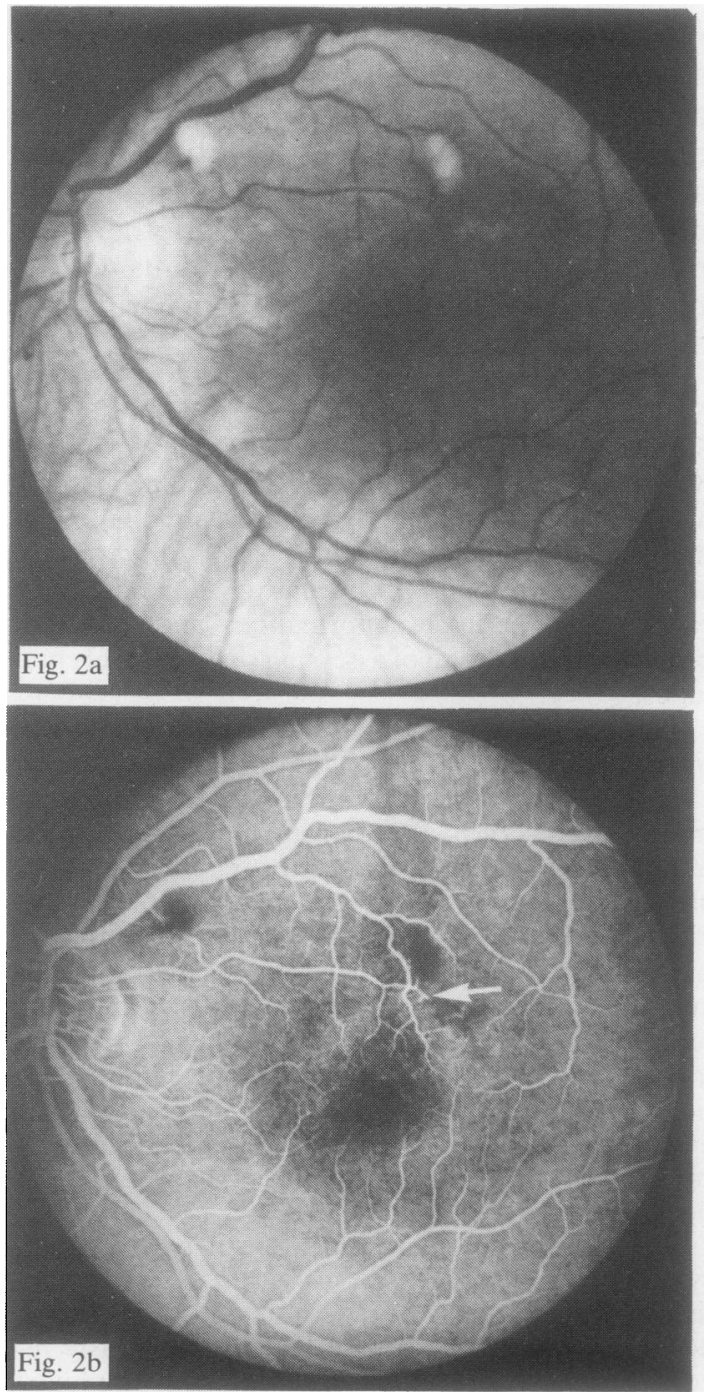

Fig. 2 Cotton-wool spots. (a) Two cotton-wool spots shown in the upper part of the photograph. (b) Fluorescein angiogram showing areas of hypofluorescence at the site of the cotton-wool spots. An occluded arteriole inferior to one cotton-wool spot is indicated by an arrow. 


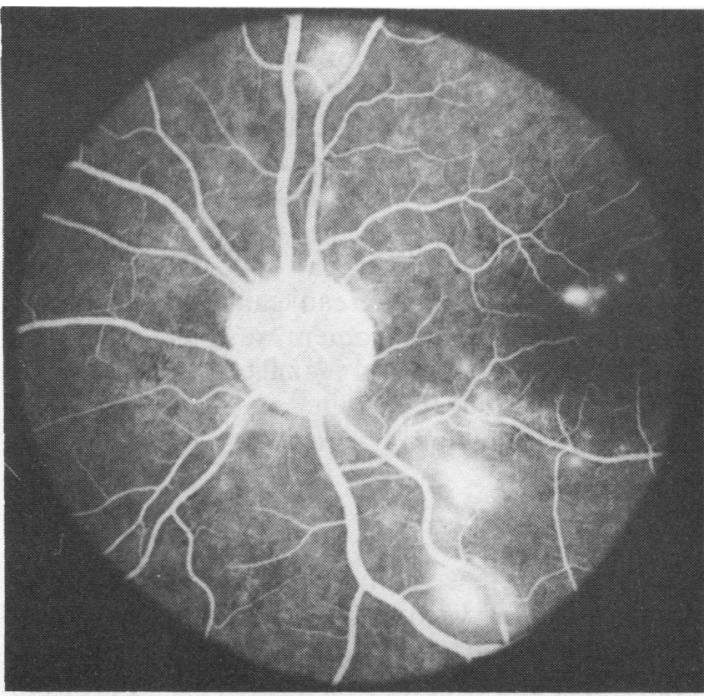

Fig. 3 Fluorescein angiogram showing focal leakage due to localised involvement of capillaries and arterioles.

these diseases rather than SLE. Two other patients with atypical retinopathy are described but not included in Tables 1 and 2.

\section{Results}

Three distinct types of retinopathy were recognised.

1. Disc vasculitis (Fig. 1)

Three patients with disc vasculitis had active disease, but only one of these had evidence of mild cerebral lupus. Marked unilateral loss of vision was recorded in 2 of the individuals with active SLE; they have subsequently developed optic atrophy. The single patient with inactive disease had disc vasculitis which was detectable only on fluorescein angiography; she suffered no loss of visual acuity.

Table 2 The relationship between the 3 forms of retinopathy and other factors which may influence retinal appearance

\begin{tabular}{|c|c|c|c|c|}
\hline & $\begin{array}{l}\text { Total } \\
(n=50)\end{array}$ & $\begin{array}{l}\text { Disc } \\
\text { vasculitis } \\
(n=4)\end{array}$ & $\begin{array}{l}\text { Cotton-wool } \\
\text { spots } \\
(n=6)\end{array}$ & $\begin{array}{l}\text { Fluorescein } \\
\text { leakage } \\
(n=5)\end{array}$ \\
\hline $\begin{array}{l}\text { Renal disease } \\
\text { Past history of } \\
\text { cerebral disease } \\
\text { Hypertension: } \\
\text { mild (BP<160/100 } \\
\text { mmHg) } \\
\text { moderate } \\
\text { BP }<160 / 100- \\
200 / 120 \mathrm{mmHg}) \\
\text { severe }(\mathrm{BP}>200 / 120 \\
\text { mmHg) } \\
\text { Diabetes }\end{array}$ & $\begin{array}{l}15 \\
12\end{array}$ & $\begin{array}{l}0 \\
0 \\
0\end{array}$ & $\begin{array}{l}0 \\
0 \\
0\end{array}$ & $\begin{array}{l}1 \\
0 \\
0\end{array}$ \\
\hline
\end{tabular}

\section{COTTON-WOOL SPOTS (Figs. 2 a, b)}

The patients with extensive cotton-wool spots all had active disease. Three of these patients had mild wellcontrolled hypertension (blood pressure below $160 / 100 \mathrm{mmHg}$ ). One had severe cerebral disease and a further patient was classed as having mild cerebral lupus manifesting as apathy and depression.
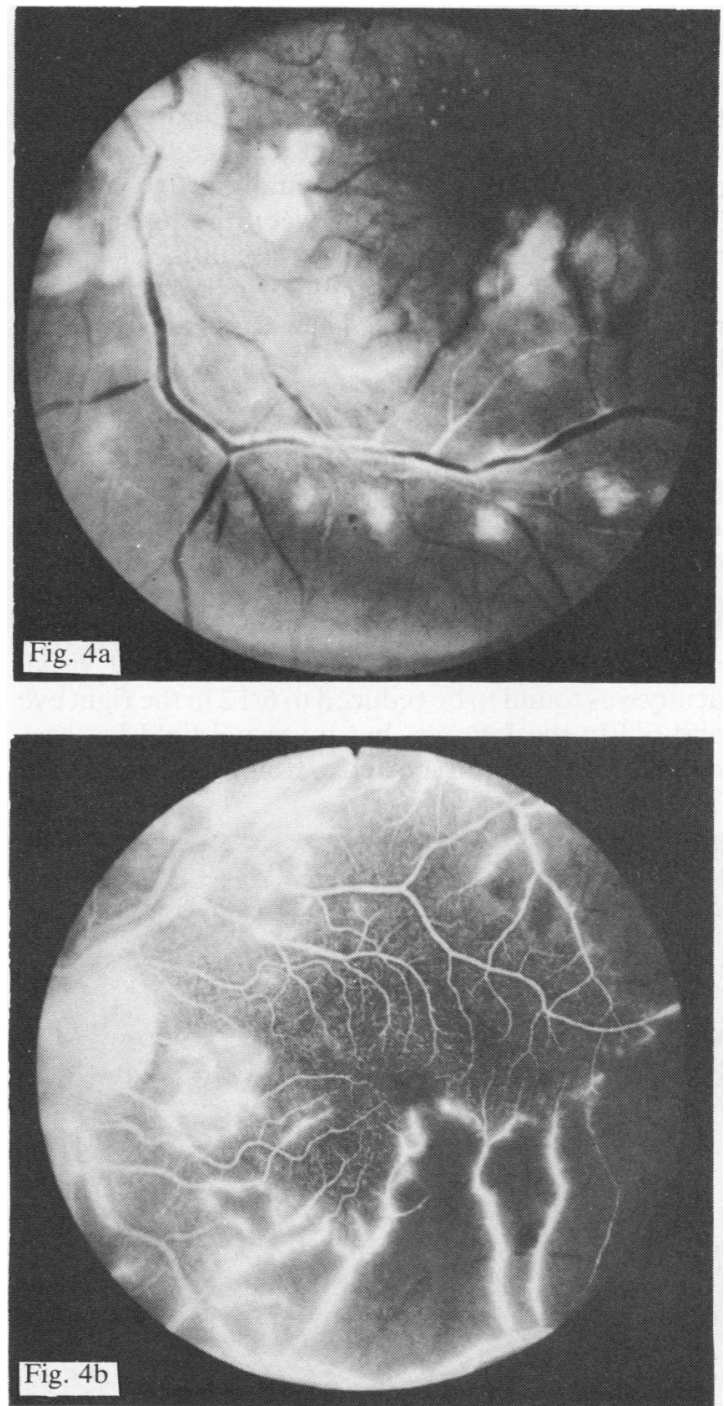

Fig. 4 (a) Atypical retinopathy of case $A$ with extensive cotton-wool spots and marked sheathing of both arteries and veins. (b) Fluorescein angiography showed extensive venous involvement with widespread leakage from the veins. Large areas of capillary nonperfusion indicate arteriolar involvement. 


\section{FOCAL LEAKAGE ON FLUORESCEIN} ANGIOGRAPHY (Fig. 3)

Cerebral disease was not a prominent feature of the patients who had focal fluorescein leakage. None of these patients were suspected of having subclinical cerebral lupus. One had severe cerebral disease and was moderately hypertensive (blood pressure $180 / 110 \mathrm{mmHg}$ ). Therefore, although fluorescein angiography identified patients with normal funduscopic appearance but abnormal vessel permeability, it failed to delineate a group of patients with either overt or subclinical cerebral disease. Whereas cotton-wool spots were restricted to those patients with active disease, fluorescein leakage occurred in patients with both active and mildly active disease. No association was found between the presence of retinal vasculitis and cutaneous vasculitis.

Two patients had retinopathy which did not conform to these three types. Their cases are reported below.

CASE A

A 24-year-old woman presented with an 18-month history of alopecia; polyarthritis, and erythematosus rash. A raised DNA binding level confirmed the diagnosis of SLE. She had intermittent mild hypertension while on steroids but never required antihypertensive medication. While her disease was quiescent she noted blurring of vision, and visual acuity was found to be reduced to $6 / 12$ in the right eye and $6 / 9$ in the left eye, but no visual field loss was detected. Both fundi showed cotton-wool spots in the

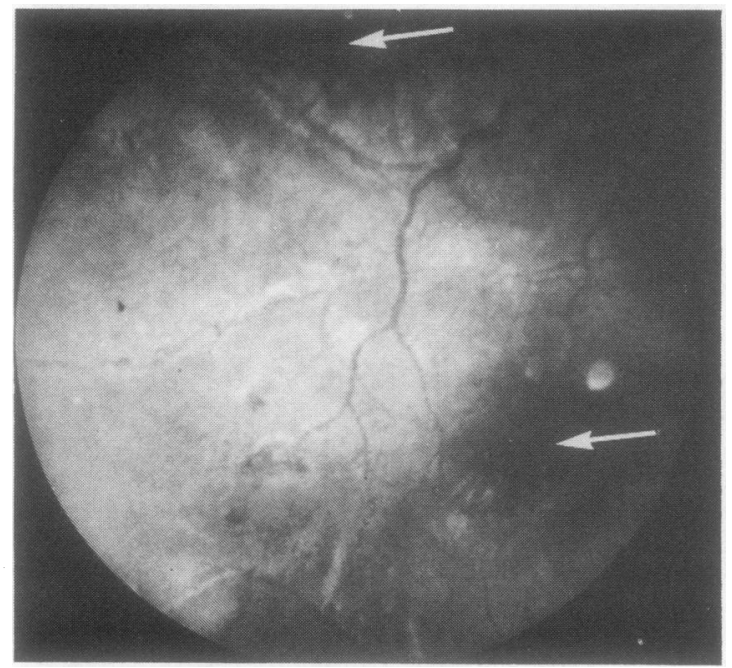

Fig. 5 Photograph of retina of case B showing arterial narrowing and irregularity with areas of new vessel formation marked by arrows. Photography poor due to lens opacities. region of the macula and extensive sheathing of both arteries and veins. This improved on treatment with corticosteroids, but a year later she had a convulsive episode in the context of mild disease activity. At this time visual acuity declined to $6 / 60$ in the right eye and $6 / 9$ in the left eye. The retinal lesions were more extensive than those seen the year before (Fig. 4a) and fluorescein angiography showed areas of hypoperfusion and widespread leakage from arteries and veins. Venous involvement was prominent with marked venous stasis and leakage (Fig. 4b). After treatment with azathioprine and steroids visual acuity and retinal appearance returned to near normal.

\section{CASE B}

This 34-year-old woman was admitted because of sudden onset of vision loss. She had a history of polyarthritis, butterfly rash, recurrent deep vein thrombosis, and spontaneous abortions. Apart from her vision loss she was asymptomatic and there were no clinical or laboratory evidence of disease activity. She was neither diabetic nor hypertensive, although she had been hypertensive during one pregnancy.

Visual acuity in the right eye was $6 / 9$ and in the left eye was perception of light only. A vitreous haemorrhage obscured any view of the left fundus. The right eye showed marked arterial narrowing, venous dilatation, and tortuosity; retinal neovascularisation was present. Fluorescein angiography demonstrated the increased permeability of the new vessels and also large areas of retinal capillary closure (Fig. 5). These findings are comparable to those reported in cases of arterial occlusive disease. ${ }^{6-9}$

\section{Discussion}

Vasculitis is believed to be the single most important process in the pathogenesis of the retinal lesions in SLE, although the retina, like the brain, rarely shows histological evidence of frank vasculitis. Goldstein and Wexler ${ }^{10}$ reported one case with extensive fibrinoid necrosis of vessel walls, but a perivascular mononuclear cell infiltrate is more common. ${ }^{11}$ Nevertheless, immunoglobulin and complement deposits have been demonstrated in the walls of the retinal vessels of a patient dying with SLE. ${ }^{12}$

There is a considerable variation in the estimated incidence of each type of retinal lesion in SLE resulting from the study of disparate groups of patients. Early studies reported a very high incidence of retinopathy; this undoubtedly reflected more severe disease at a time when treatment was less effective and mortality rates could exceed $30 \%{ }^{13}$ Retinal haemorrhages and cotton-wool spots were each reported in one early study to occur in $28 \%$ of 
patients, ${ }^{13}$ whereas more recently cotton-wool spots were found in only 2 of 61 patients studied and retinal haemorrhages were not seen at all. ${ }^{14}$ These patients, however, were adequately treated outpatients. Many of the studies include unspecified numbers of patients with severe renal disease, diabetes, and hypertension. ${ }^{13}{ }^{15}$ As a result a wide range of retinal abnormalities have come to be regarded as manifestations of SLE. They include focal or generalised arteriolar narrowing, superficial flame-shaped or preretinal haemorrhages, arteriovenous nipping, venous engorgement, and retinal detachment. ${ }^{713} 15$ These lesions may occasionally be seen in patients with severe disease, but they are not regular features of SLE retinopathy. The present study suggests that 3 relatively distinct types of SLE retinopathy can be discerned. Unusual exceptions are described in section 4 below.

1. Extensive cotton wool spots. Cotton-wool spots correspond to focal areas of ischaemia, producing swelling within the nerve fibre layer of the retina. ${ }^{16}$ They result from an accumulation of axoplasmic material following the interruption of axoplasmic transport. ${ }^{17}{ }^{18}$ The ischaemia is thought to be a consequence of retinal vasculitis involving primarily the retinal arterioles, although frequently in SLE prominent dilatation of these arterioles is observed. This is in sharp contrast to the other retinopathies with extensive cotton-wool spot formation such as hypertension and diabetes, where the retinal arterioles are often attenuated and may become occluded; these diseases are marked by a greater degree of ischaemia than in SLE. Usually in hypertension and diabetes there is extensive nonperfusion of retinal capillaries, which is best seen on fluorescein angiography. Nevertheless, when diabetes or hypertension coincides with SLE, the relative contribution of each can be difficult to assess.

2. Disc vasculitis. This refers to unilateral optic disc swelling resulting from vasculitis affecting vessels within the optic nerve (ischaemic optic neuropathy). The appearances are due to an accumulation of axoplasmic material within the optic nerve. ${ }^{17}$ Symptoms may resemble those of optic neuritis, but in ischaemic optic neuropathy the field loss, instead of being patchy or central, is either complete or is an altitudinal hemianopia. Visual acuity can be reduced in both conditions, but, whereas in optic neuritis there is almost always considerable subsequent recovery of vision, in clinically obvious disc vasculitis such improvement is unusual.

In the present study 2 patients had disc vasculitis of sufficient severity to give rise to vision loss. Two other patients had only unilateral leakage from disc vessels on fluorescein angiography and no visual symptoms. Fluorescein angiography proved there- fore to be a sensitive means of detecting mild disc vasculitis. Although the importance of detecting such subclinical disc vasculitis is uncertain, these 2 patients suggest this condition to be much more benign, as neither progressed to overt disc vasculitis and vision loss. Evidence of disc vasculitis may persist for some time, and this may explain the occurrence of disc vasculitis in the patient with inactive disease. Alternatively, retinal vasculitis may be the sole manifestation of disease activity. ${ }^{10}$

3. Focal leakage on fluorescein angiography. These patients have no visual complaints, and their fundi appear normal on fundoscopy. Fluorescein angiography, however, reveals focal capillary vasculitis; minimal focal arteriolar involvement may also be present. This study suggests that these findings connote active disease but not necessarily very active disease or cerebral involvement.

4a. Arterial occlusive disease. Arterial occlusive disease is a rare but distinct entity in SLE. ${ }^{6-9}$ Severe widespread retinal ischaemia occurs and vision loss may follow. In this series we report a patient (case B) with widespread arteriolar occlusions who developed neovascularisation complicated by vitreous haemorrhage. Neovascularisation is not generally regarded as a feature of SLE retinopathy. It is triggered by severe retinal ischaemia and probably occurs in SLE only as a rare complication of arterial occlusive diseases. Generally, arterial involvement in SLE does not produce such extensive retinal ischaemia.

4b. Retinal vein disease. The retinopathy of SLE is not primarily a venous disease. However, venous stasis and venous engorgement may occur, ${ }^{79}$ and there have been occasional reports of central retinal vein occlusion. ${ }^{19}{ }^{20}$ One patient in this study (case $A$ ), with active cerebral disease, had extensive venous stasis and widespread leakage of fluorescein from the veins. This appearance was very similar to that of Behcet's disease, but multiple cotton-wool spots were also present, indicating coexistent arterial involvement (Fig. 4a).

4c. Retinal haemorrhages. This study includes only one patient with retinal haemorrhages. She had mild hypertension $(160 / 90 \mathrm{mmHg})$ of short duration with dilatation of the retinal arterioles (Fig. $2 a$ and b); her retinal lesions were probably a consequence of her SLE rather than of her hypertension. In our experience retinal haemorrhages are unusual in SLE in the absence of hypertension, diabetes, or atypical SLE retinopathy such as retinal vein thrombosis or arterial occlusive disease, although they are occasionally seen in advanced disease.

VISION LOSS DUE TO SLE RETINOPATHY The prognosis for vision is good in most types of SLE retinopathy. Even extensive cotton-wool spots due to 
SLE do not generally cause significant vision loss, in contrast to other diseases producing the same lesions. This is perhaps a consequence of the more severe retinal ischaemia caused by diabetic or hypertensive lesions. Disc vasculitis and arterial occlusive disease are the 2 forms of SLE retinopathy which may lead to permanent loss of vision. Widespread retinal ischaemia may cause blindness in arterial occlusive disease, and vision loss may result from ischaemia of the optic nerve in disc vasculitis.

Our findings are at variance with those of an earlier study of fluorescein angiography in SLE in which $18 \%$ of patients were found to have microaneurysms and $12 \%$ fluorescein leakage in the absence of funduscopic abnormalities. ${ }^{21}$ No microaneurysms were detected in our patients. We do not regard microaneurysms as a regular feature of SLE retinopathy. On fluorescein angiography either focal fluorescein leakage or an end-on view of a vessel may give the appearance of a microaneurysm.

This study confirms earlier observations ${ }^{14}$ that retinopathy in general is a feature of more active disease. Cotton-wool spots in particular are associated with disease activity. Nevertheless, one of our patients had disc vasculitis while their disease was inactive. The history of case $B$, and occasional published reports, suggest that severe and widespread retinal vasculitis can occur without evidence of disease activity elsewhere. ${ }^{1021}$ The general experience in SLE is that disease activity may manifest in only one organ or system, and thus the retina appears to be no different from other organs in this regard.

This study does not confirm that fluorescein angiography assists in the evaluation of patients with SLE. Firstly, although focal fluorescein leakage is associated with disease activity, it does not signify very active disease or cerebral lupus. Secondly, the retinal lesions are not diagnostic of SLE. And, thirdly, vasculitis in the retina does not necessarily mirror vasculitis in other organs such as the brain and skin.

We acknowledge the assistance of Mr Michael Sanders, who referred case $A$.

\section{References}

${ }^{1}$ Edmonds J P, Bruneau C, Hughes G R V. Assessment of activity in SLE: a clinical and serological study. (Abst). Ann Rheum Dis $1975 ; 34: 543-4$.

2 Appenzeller O, Williams R C. Cerebral lupus erythematosus. Ann Intern Med 1979; 90: 430-1.

${ }^{3}$ Gibson T, Myers A R. Nervous system involvement in systemic lupus erythematosus. Ann Rheum Dis 1976; 35: 402-6.

4 Baker M. Psychopathology in systemic lupus erythematosus. I. Psychiatric observations. Semin Arthritis Rheum 1973; 3: 95-110.

5 Goodwin J S, Goodwin J M. Cerebritis in lupus erythematosus. Ann Intern Med 1979; 90: 437-8.

- Gold D H, Feiner L, Henkind P. Retinal arterial occlusive disease in systemic lupus erythematosus. Arch Ophthalmol 1977; 95: 1580-5.

7 Bishko F. Retinopathy in systemic lupus erythematosus. Arthritis Rheum 1972; 15: 57-63.

- Coppeto J, Lessel S. Retinopathy in systemic lupus erythematosus. Arch Ophthalmol 1977; 95: 794-7.

- Pfaffenbach D D, Hollenhorst R W. Microangiography of the retinal arterioles. JAMA 1973; 225: 480-3.

${ }^{10}$ Goldstein I, Wexler D. Retinal vascular disease in a case of acute lupus erythematosus disseminatus Arch Ophthalmol 1932; 8: 852-7.

11 Aiello J S. Ocular findings in lupus erythematosus. Am J Ophthalmol 1952; 35: 837-43.

12 Aronson A J, Ordonez N G, Diddie K R, Ernest J T. Immunecomplex deposition in the eye in systemic lupus erythematosus. Arch Intern Med 1979; 139: 1312-3.

13 Shearn M A, Pirofsky B. Disseminated lupus erythematosus. Arch Intern Med 1952; 90: 790-807.

${ }^{14}$ Gold D M, Morris D A, Henkind P. Ocular findings in systemic lupus erythematosus. Br J Ophthalmol 1972; 56: 800-4.

15 Armas-Cruz R, Harnecker J, Ducach G, Jalil J, Gonzalez F. Clinical diagnosis of systemic lupus erythematosus. Am J Med 1958; 25: 409-19.

${ }^{16}$ Patz A. Retinal vascular diseases. $N$ Engl J Med 1978; 298: $1451-4$.

${ }^{17}$ McLeod D, Marshall J, Kohner E M. Role of axoplasmic transport in the pathology of ischaemic disc swelling. BrJ Ophthalmol 1980; 64: 247-61.

${ }^{18}$ McLeod D, Marshall J, Kohner E M. Pathogenesis of cotton-wool spots. Br J Ophthalmol 1977; 61: 177-91.

19 Silverman M, Lubeck M J, Briney W G. Central retinal vein occlusion complicating systemic lupus erythematosus. Arthritis Rheum 1978; 21: 839-43.

${ }^{20}$ Harvey A M, Shulman L E, Tumutty P A, Conley C L, Schoenrich E H. Systemic lupus erythematosus: review of the literature and clinical analysis of 138 cases. Medicine (Baltimore) 1954; 33: 291-437.

${ }^{21}$ Santos R, Barojas E, Alarcon-Segovia D, Ibanez G. Retinal microangiography in systemic lupus erythematosus. Am J Ophthalmol 1975; 80: 249-52. 